\title{
Business Security Design Improvement Using Digitization
}

\author{
Prof. Ivan Zellar \\ Management B School, Russia \\ ivamzellar@mail.ru
}

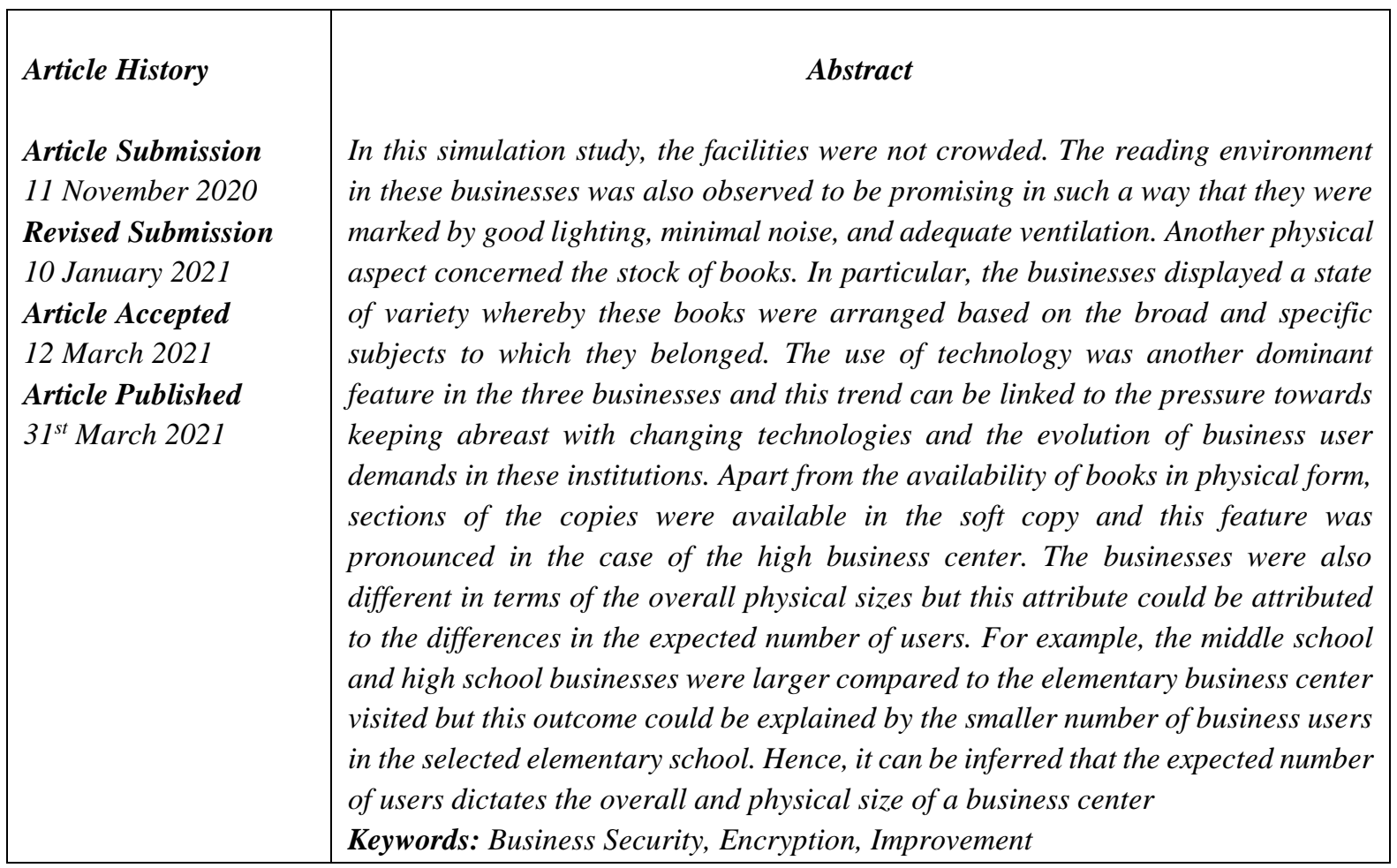

\section{Introduction}

According to Rumsey (2008), businesses are no longer places established for the sole purpose of storing and distributing books. In addition, these places are no longer restricted to studying. Instead, technological changes have prompted alterations in business operations. As affirmed by Shumaker (2011), these places have continually adapted by assuming a people-centric approach, rather than a book-centric path. The eventuality is the businesses in settings such as elementary, middle, and high schools have emerged as community resources for making, creating, and collaborating. Some of the areas or facilities that the design process needs to consider include computers, meeting places, audio/visual labs, media storage, book storage or shelves, and the outdoor seating area. Others include the indoor seating area, bins for recycling, a small office for the business staff, and the librarian's desk (Rumsey, 2008). This study's central aim was to conduct a computer network simulation exercise to design a business center media center. The motivation was to predict how computer network processes might inform the future of structural designs.

Based on the insights gained from the context of elementary, middle, and high business center designs and requirements, mixed outcomes are observed. In particular, these facilities are observed to exhibit differences but parallels can also be drawn relative to their designs and requirements. From a personal survey, common characteristics associated with the design of businesses in the three institutions revealed several types of space. These broad types included special use space, meeting space, staff work space, and user seating space. Others included the electronic workstation space and the collection space. However, computer workstation clusters were dominant in the high school 
compared to the elementary and middle school businesses. On the other hand, collaborative learning spaces dominated the context of the elementary business center, including the presence of gaming environments that were deemed suitable for the learning stage of the students or facility's users Similarly, the high business center was marked by the presence of a multimedia presentation center or presentation support center in the form of an electronic classroom. Hence, there was a marked difference between the three sets of businesses and these differences could be attributed to the learning stage of the students in those institutions. Despite the variations, the common theme observed was that the three businesses were designed in such a way that they reflected privacy and personalized study areas.

\section{Methodology}

Zone planning formed another aspect of consideration. In particular, the businesses exhibited notable similarities in the design and requirements, with zone planning in focus. For instance, the design of the three businesses indicated that the librarian's desk is located next to the entrance and the bag store and display boards would follow. Whereas the high business center had the bag store next to the librarian's desk while the display board was in the opposite direction but still at the entrance, the elementary and middle school businesses had the librarian's desk, bag store, and display board on the same side and in that order. The zone planning assessment revealed further that the materials or spaces that addressed common themes or were complementary to each other were located in the same direction. For instance, the three businesses had the classroom collaboration space and the teacher's resources located next to each other. A similar arrangement was observed regarding the media production space and social networking gaming, the staff offices, work space, and the circulation desk, as well as the fiction and non-fiction sections. It is also worth noting that in the three businesses, most of the spaces and facilities mentioned above were located towards the extremes or surrounding walls of the businesses. Hence, the central part of the businesses was used as a space for studying, teaching, reading, viewing, and listening. The difference in these arrangements was that the high business center dedicated part of the central space for purposes of browsing and collaboration.

\section{Results and Discussion}

From the perspective of the simulated physical environment and business environment, the three businesses were observed to exhibit various common characteristics. For instance, the work areas were sufficient with book stacks organized in a way that enables the users to access them with ease, complemented by plenty of light to work and read. However, special activity corners were observed to be dominant in the elementary business center compared to the case of the middle and high school businesses. In the elementary school institution, the special activity corner had a games corner, the author's corner, and the children's corner from which educational activities would be conducted, reflecting an engaging environment, decorations and displays were also observed to dominate the elementary and middle school businesses, compared to the physical space and environment of the high business center. Particularly, the decorations and displays sought to reinforce academic content and reflect the national, as well as local culture.

An additional component, as deduced from the business design above, concerns universality. In particular, the student community could entail learners with mixed abilities and needs, with those requiring special needs inclusive. Therefore, the need to assure inclusivity forms a crucial component of an ideal business design. In the design above, the audio-visual room seeks to cater for the needs of different facility users, assuring universality. A specific example is a case in which learners are expected to attend sessions involving PowerPoint presentations. In such a case, the audio-visual room becomes appropriate and is expected to be included in the floor design. On the other hand, some situations could 
involve learners with special needs, including the visually impaired groups. With the presence of the audio-visual room and its associated facilities, the needs of such learners can be met with ease; a trend that reflects the universality of the designed business.

\section{Conclusion}

Overall, the computer network simulation exercise suggests that major issues to consider in a business design are seen to entail inclusivity, the ease of movement, and the predicted number of users in the respective sections (for purposes of ample space allocation).

\section{References}

1. Rumsey, M. (2008).Foreign and international law librarianship.Legal Reference Services Quarterly, 25, 73-88

2. Shumaker, D. (2011). "Special Businesses." In Encyclopedia of Business and Information Sciences ( $3^{\text {rd }}$ Ed.), 4966-4974. New York: Taylor and Francis

3. Guerra, E., de Lara, J., Kolovos, D., Paige, R.: A visual specification language for model-to-model transformations. In: IEEE Symposium on Visual Languages and Human-Centric Computing (2010).

4. ISO: ISO/IEC 27000 Information technology-Security techniques-Information security management systems - Overview and vocabulary. Technical report (2014)

5. Kalloniatis, C., Kavakli, E., Gritzalis, S.: Using privacy process patterns for incorporating privacy requirements into the system design process. In: 2nd International Conference on Availability, Reliability and Security (ARES'07), pp. 1009-1017. IEEE, Washington (2007).

6. Kalloniatis, C., Kavakli, E., Gritzalis, S.: Addressing privacy requirements in system design: the pris method. Requir. Eng. 13(3), 241-255 (2008)

7. Kienzle, D.M., Elder, M.C.: Security patterns for web application development. University of Virginia technical report (2002)

8. Lavérdiere, M., Mourad, A., Hanna, A., Debbabi, M.: Security design patterns: survey and evaluation. In: 2006 Canadian Conference on Electrical and Computer Engineering, pp. 1605-1608. IEEE, Washington (2006)

9. Leitner, M., Miller, M., Rinderle-Ma, S.: An analysis and evaluation of security aspects in the business process model and notation. In: 8th International Conference on Availability, Reliability and Security (ARES'13), pp. 262-267. IEEE, Washington (2013)

10. Li, T., Paja, E., Mylopoulos, J., Horkoff, J., Beckers, K.: Security attack analysis using attack patterns. In: 2016 IEEE 10th International Conference on Research Challenges in Information Science (RCIS), pp. 1-13. IEEE, Washington (2016) 